\title{
Z-Axis Voxel Dimension
}

National Cancer Institute

\section{Source}

National Cancer Institute. Z-Axis Voxel Dimension. NCI Thesaurus. Code C156499.

The linear measurement of a voxel along the Z-axis. 